\title{
Sostenibilidad de las capitales de provincia de España
}

\author{
Francisco RIVERO PALLARÉS ${ }^{1}$ \\ friverop@hotmail.com \\ Josefa María RodRíGUEz MELlado ${ }^{2}$ \\ josefarm7@hotmail.com
}

Recibido: 29 de febrero del 2012

Enviado a evaluar: 5 de marzo del 2012

Aceptado: 27 de julio del 2012

\section{RESUMEN}

Se establecen los valores óptimos para los indicadores recogidos en la publicación "Sostenibilidad local: una aproximación urbana y rural" del Observatorio de Sostenibilidad de España, y, más concretamente, en el capítulo "Guía de aplicación para un sistema integrado de indicadores urbanos" desarrollado por el Observatorio de Medio Ambiente Urbano de Málaga (OMAU) junto con la oficina de cooperación de la Comisión Europea (EUROPEAID) y Naciones Unidas (UNHABITAT). Se define y aplica una función de sostenibilidad a los citados indicadores y se obtiene el valor de sostenibilidad de las capitales de provincia de España. Los valores de sostenibilidad alcanzados por las ciudades españolas son bajos para la mayoría de los indicadores, al menos con las premisas consideradas en este trabajo.

Palabras clave: Función de sostenibilidad, residuos urbanos, consumo de agua, consumo de energía eléctrica, zonas verdes, PM10, tasa de paro, motorización, viviendas, densidad urbana.

\section{Sustainability of the provincial capitals of Spain}

\begin{abstract}
It is established the optimum values for the indicators contained in the publication "local sustainability: a rural and urban approach" of Sustainability Observatory of Spain, and, more specifically, in the chapter "Application guide for an integrated system of urban indicators" developed by the Observatory of Urban Environment of Malaga (OMAU) together with the Office of Cooperation of the European Commission (EUROPEAID) and the United Nations (UNHABITAT). It is defined and applied a function of sustainability to the above-mentioned indicators, and gets the value of sustainability of the capital cities of Spain. The values of sustainability obtained by the Spanish cities are low for most of the indicators, at least with the premises referred to in this work.

\footnotetext{
1 Francisco Rivero Pallarés es investigador en Sostenibilidad.

2 Josefa María Rodríguez Mellado es Consultora Independiente especialista en Medio Ambiente y Sostenibilidad.
} 
Keywords: Function of sustainability, municipal waste, water consumption, electric energy consumption, parkland, PM10, unemployment rate, motorization, housing, population density.

\section{Durabilité des capitales provinciales de l'Espagne}

\section{RESUMÉ}

On établée les valeurs optimales pour les indicateurs figurant dans la publication "durabilité locale: une approche rurale et urbaine" de Durabilité Observatory de l'Espagne et, plus précisément, dans le chapitre «Guide de demande pour un système intégré d'indicateurs urbains» élaboré par l'Observatoire de l'Environnement Urbain de Malaga (OMAU) avec le Bureau de la Coopération de la La Commission Européenne (EUROPEAID) et l'Organisation des Nations Unies (ONU-Habitat). On définé et appliqué une fonction de durabilité aux indicateurs susmentionnés et obtient la valeur de la durabilité des capitales de la province de l'Espagne. Les valeurs du développement durable par les villes espagnoles sont faibles pour la plupart des indicateurs, au moins avec les locaux visés dans ce travail.

Mots clé: Fonction of durabilité, déchets municipaux, consommation d'eau, consommation d'énergie électrique, parc, PM10, taux de chômage, motorization, logement, densité de population.

\section{INTRODUCCIÓN}

Los análisis de sostenibilidad realizados hasta el momento no presentan una metodología y unos criterios objetivos que permitan comparar la situación de dos ámbitos de estudio o de dos indicadores distintos.

Los autores han presentado varios trabajos (Rodríguez Mellado y Rivero Pallarés, 2010a y 2010b) en los que establecen una metodología basada en una serie de postulados que permiten estandarizar los valores alcanzados por los diferentes indicadores de sostenibilidad y realizar un diagnóstico cuantificado sobre el estado de sostenibilidad en que se encuentra un determinado sistema (ciudad, país, actividad económica, etc.). Así mismo se definen los valores que deben alcanzar los diferentes indicadores para conseguir una sostenibilidad máxima (valores óptimos). Esta metodología hace posible el establecimiento de líneas de actuación prioritarias acordes con las necesidades detectadas en cada zona de estudio.

El objetivo de este trabajo es seguir en esta línea de definir una función de sostenibilidad que permita la estandarización de los valores de sostenibilidad alcanzados por los diferentes indicadores. El presente trabajo es una aplicación a un conjunto de datos presentados en la publicación "Sostenibilidad local: una aproximación urbana y rural" del Observatorio de Sostenibilidad de España, y, más concretamente, en el capítulo "Guía de aplicación para un sistema integrado de indicadores urbanos" desarrollado por el Observatorio de Medio Ambiente Urbano de Málaga 
(OMAU) junto con la oficina de cooperación de la Comisión Europea (EUROPEAID) y Naciones Unidas (UNHABITAT).

\section{METODOLOGÍA}

\subsection{FUNCIÓN DE SOSTENIBILIDAD}

En los trabajos reseñados de los autores se deducen unas funciones de sostenibilidad sensu stricto (1) y sensu lato (2) que vienen dadas por las expresiones

$$
\begin{aligned}
& S=e^{-9,9035 \cdot\left(\frac{l-10}{10}\right)^{2}} \\
& S=e^{-5,3 \cdot\left(\frac{1-10}{10}\right)^{2}}
\end{aligned}
$$

La figura 1 muestra el aspecto de la función de sostenibilidad considerando ambas expresiones.

Figura 1. Representación gráfica de la función de sostenibilidad sensu stricto (línea azul) y sensu lato (línea roja)

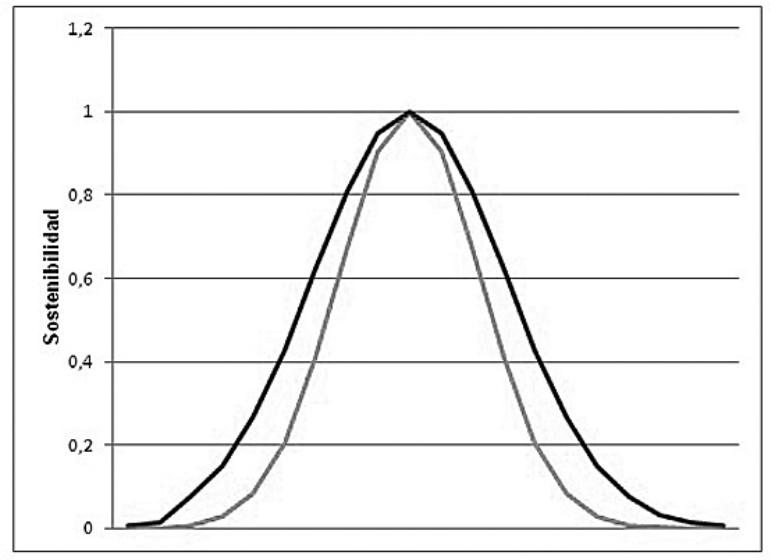

Esta gráfica muestra cómo el valor de sostenibilidad presenta un máximo cuando el valor del indicador es $\mathrm{I}_{\mathrm{O}}$, $\mathrm{y}$ cómo la sostenibilidad va disminuyendo conforme el valor del indicador se aleja de este valor $\mathrm{I}_{\mathrm{O}}$ tanto hacia la derecha como hacia la izquierda.

El indicador puede estar definido de tal modo que se use sólo la rama de la izquierda o la rama de la derecha de la función. El primer caso se produce si la sostenibilidad varía entre cero y uno cuando $\mathrm{I}<\mathrm{I}_{\mathrm{O}}$, y la sostenibilidad permanece con el valor de 1 para valores de $\mathrm{I}>\mathrm{I}_{\mathrm{O}}$, mientras que el segundo caso se produce si el indi- 
cador alcanza la sostenibilidad de 1 cuando $\mathrm{I}<\mathrm{IO}$, y la sostenibilidad disminuye para valores de $\mathrm{I}>\mathrm{I}_{\mathrm{O}}$.

Puede plantearse la situación que un determinado indicador tenga como valor óptimo cero, es decir que la sostenibilidad sea máxima cuando Io $=0$. En este caso hay que definir un valor máximo del indicador $\left(\mathrm{I}_{\mathrm{M}}\right)$ que produce una sostenibilidad de cero. Se demuestra que las expresiones (1) y (2) se transforman, respectivamente, en las siguientes expresiones:

$$
\begin{aligned}
& S=e^{-9,9035 \cdot\left(\frac{l}{M M}\right)^{2}} \\
& S=e^{-5,3 \cdot\left(\frac{l}{I M}\right)^{2}}
\end{aligned}
$$

\subsection{APLICACIÓN A LOS DATOS DE LA GUÍA DE APLICACIÓN PARA UN SISTEMA INTEGRADO DE INDICADORES URBANOS}

La Guía de aplicación para un sistema integrado de indicadores urbanos define una serie de indicadores de sostenibilidad y recoge los valores que alcanzan dichos indicadores en las diferentes capitales de provincia. A continuación se analizan estos indicadores y se definen los valores de IO o de IM para aquellos que pueden medirse. El nombre de cada indicador va acompañado de unas iniciales en mayúsculas que permiten identificarlo en posteriores citas en este documento.

\section{Generación de residuos urbanos (GRU)}

Se mide en Kg. residuos/habitante y día ( $\mathrm{kg} / \mathrm{hab}$ día)

Se considera que los residuos urbanos (RU a partir de ahora) son la materia no aprovechada al pasar de un nivel trófico a otro en los ecosistemas. Si se aplica la regla del $10 \%$, la cantidad de residuos debe ser la indicada en la siguiente figura:

Figura 2. Distribución de las masas según la regla del 10 \%. Elaboración propia

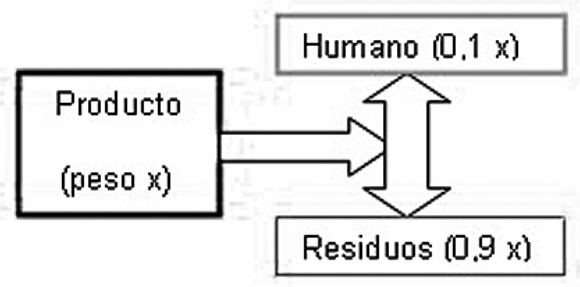

Se concluye que la relación peso RU/peso humano debe ser de 9. Es decir, un humano debe producir nueve veces su peso en RU para que sea ecológicamente sostenible. Hay que conocer el peso medio de los ciudadanos, que denominaremos Pm. El peso medio se ha obtenido combinando los pesos teóricos que deben tener las personas según edad y sexo y la distribución de edades y sexo de cada ciudad. Hay que 
considerar la materia seca, que se acepta es un $30 \%$ en el cuerpo humano. Por lo tanto se concluye que el valor que se considera óptimo para este indicador es:
Io $=9 \times 0,3 \times \mathrm{Pm} / 365$
es decir:
Io $=0,0074 \times \mathrm{Pm}$

Que viene dado en $\mathrm{kg} / \mathrm{hab}$ día, por lo que la función de sostenibilidad queda

SGRUSS $=e^{-9,9035 \cdot\left(\frac{(-0,0074 \cdot P m}{0,0074 \cdot P m}\right)^{2}} \quad$ (7) ó

SGRUSl $=e^{-5,3 \cdot\left(\frac{I-0,0074 \cdot P m}{0,0074 \cdot P m}\right)^{2}}$

para valores de GRU $>0,0074$ x Pm. Para valores de GRU $<0,0074$ x Pm se considera que la sostenibilidad es igual a 1 .

SGRUss y SGRUsl representan la sostenibilidad para la producción de residuos sensu stricto y sensu lato respectivamente

Tal y como está definido el indicador se considera sólo la rama derecha de la función de sostenibilidad.

\section{Consumo de agua en los hogares (CAH)}

Se mide en litros/habitante y día (1/hab día).

Se considera que el agua disponible es la diferencia entre la precipitación $(\mathrm{P})$ y la evapotranspiración (ET) y viene dado en $\mathrm{mm}$, o, lo que es lo mismo, $1 / \mathrm{m}^{2}$. Es decir:

$\mathrm{A}_{\text {dis }}=\mathrm{P}-\mathrm{ET}$

La evapotranspiración se calcula según el método de Thornthwaite según la expresión:

$E T=1,6 *\left(\frac{10 * t}{l}\right)^{a}$

Donde: -ET es la evapotranspiración potencial $(\mathrm{mm} / \mathrm{mes})$

-t es la temperatura media mensual, medida en grados centígrados

-I es el índice de calor anual

-a se obtiene según la expresión:

$\mathrm{a}=0,492+0,0179 * \mathrm{I}+0,0000771 * \mathrm{I} 2+0,000000675^{*} \mathrm{I}^{3}$

El índice de calor anual se calcula a partir de las temperaturas medias de los doce meses según la expresión:

$I=\sum_{i=1}^{12}\left(\frac{t i}{5}\right)^{1,5}$

Donde ti es la temperatura media del mes i.

Hay que definir una superficie que recoja el agua disponible. Este trabajo considera que esta superficie debe ser la superficie urbana que debería tener la ciudad 
supuesto que es totalmente sostenible. Una ciudad puede tener una gran superficie urbana porque tiene una baja densidad de viviendas, situación sobre la que hay un común acuerdo que no es demasiado sostenible. Hay que considerar la superficie que debería tener la ciudad si tuviera una densidad de viviendas sostenible. Más adelante se define un indicador que se denomina Densidad Urbana. En dicho indicador se razona que un valor óptimo puede ser 217,5 hab/Ha. Para deducir cual es la superficie que debería tener la ciudad se realiza el cociente:

$\mathrm{S}_{\mathrm{U}}{ }^{\prime}=$ Total habitantes / 217,5 hab/Ha

Que viene dado en hectáreas. El total de litros de agua disponible será:

Total agua $=\mathrm{A}_{\text {dis }} * \mathrm{SU} * 10^{4}$

El valor óptimo del indicador viene dado por la expresión:

Io $=\left(\mathrm{A}_{\mathrm{dis}} * \mathrm{SU} * 10^{4}\right) /($ Total habitantes $* 365)(14)$

Que queda: $\quad$ Io $=0,126 * \mathrm{~A}_{\text {dis }}$

Que viene dado en $1 /$ hab día, por lo que la función de sostenibilidad queda

SCASS $=e^{-9,9035 \cdot\left(\frac{I-0,126 \cdot \text { Adis }}{0,126 * \text { Adis }}\right)^{2}}$

SCAsl $=e^{-5,3 \cdot\left(\frac{l-0,126 \cdot \text { Adis }}{0,126 * \text { Adis }}\right)^{2}}$

Para valores de $\mathrm{CA}>0,126 * \mathrm{~A}_{\text {dis }}$. Para valores de $\mathrm{CA}<=0,126 * \mathrm{~A}_{\text {dis }}$ se considera que la sostenibilidad es 1 .

Donde SCAss y SCAsl representan la sostenibilidad del consumo de agua sensu stricto y sensu lato respectivamente

Tal y como está definido el indicador se considera sólo la rama derecha de la función de sostenibilidad

\section{Consumo de energía eléctrica por habitante estimado (CEHe)}

Se mide en Megavatioshora/habitante y año (Mwh/hab año)

Sea Eh la cantidad de kilocalorías necesarias para el mantenimiento del cuerpo humano en un día. Para transformarlas en Mwh/hab año se tiene:

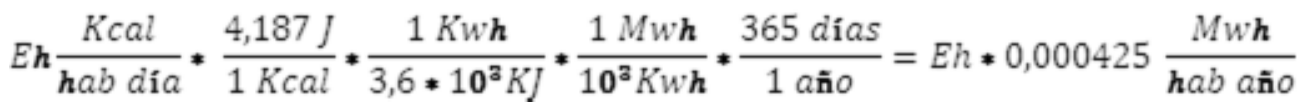

Si consideramos la regla del $10 \%$, la energía que necesita una persona para que sea ecológicamente sostenible será

Io $=\mathrm{Eh} * 0,00425 \mathrm{Mwh} / \mathrm{hab}$ año

El valor de Eh se ha obtenido combinando los valores energéticos que necesitan las personas según edad y sexo y la distribución de edades y sexo de cada ciudad. La función de sostenibilidad queda: 
SCEHeSS $=e^{-9,9035 \cdot\left(\frac{I-E h \cdot 0,00425}{E h \cdot 0,00425}\right)^{2}}$

SCEHeSl $=e^{-5,3 \cdot\left(\frac{l-E h \cdot 0,00425}{E h \cdot 0,00425}\right)^{2}}$

para valores de $\mathrm{CEHe}>\mathrm{Eh} * 0,00425 \mathrm{Mwh} / \mathrm{hab}$ año. Para valores de $\mathrm{CEHe}<\mathrm{E}_{\mathrm{h}}{ }^{*} 0,00425 \mathrm{Mwh} / \mathrm{hab}$ año se considera que la sostenibilidad es igual a 1

Donde SCEHess y SCEHesl representan la sostenibilidad del consumo de energía sensu stricto y sensu lato respectivamente

Tal y como está definido el indicador se considera sólo la rama derecha de la función de sostenibilidad.

Los datos que aporta el estudio sobre energía consumida en un año son: energía eléctrica $(\mathrm{Mwh} / \mathrm{hab})$, gases licuados del petróleo $(\mathrm{Tm} / \mathrm{hab})$, gas natural (Gcal/hab), hidrocarburos totales (Tep/hab) y Renovables (Tep/100 hab). Para calcular el consumo total de energía se transforman estas unidades a Mwh/Hab año.

\section{Superficie de zonas verdes ( $\mathrm{ZV})$}

Se mide en metros cuadrados por habitante $(\mathrm{m} 2 / \mathrm{hab})$

La OMS recomienda el intervalo $10-15 \mathrm{~m} 2 / \mathrm{hab}$. El estándar europeo deseable es de $25 \mathrm{~m} 2 / \mathrm{hab}$. Se considera Io $=20 \mathrm{~m} 2 / \mathrm{hab}$. La función de sostenibilidad queda:

SZVSS $=e^{-9,9035 \cdot\left(\frac{l-20}{20}\right)^{2}}$

$S Z V s l=e^{-5,3 \cdot\left(\frac{l-20}{20}\right)^{2}}$

(21) ó

Para valores de $\mathrm{SZV}<20 \mathrm{~m} 2 / \mathrm{hab}$. Para valores de $\mathrm{SZV}>20 \mathrm{~m} 2 / \mathrm{hab}$ se considera que la sostenibilidad es igual a 1.

Donde SZVss y SZVsl representan la sostenibilidad para la superficie de zonas verdes sensu stricto y sensu lato respectivamente

Tal y como está definido el indicador se considera sólo la rama izquierda de la función de sostenibilidad.

\section{Concentración de PM10 (PM10)}

Se mide en $\mu \mathrm{g}$ PM10/m3 de aire. El parámetro más utilizado es el número de días, en un año, en que se supera la concentración de $50 \mu \mathrm{g} \mathrm{PM10/m3} \mathrm{de} \mathrm{aire.} \mathrm{Se}$ considera que el valor ideal es cero días $(\mathrm{Io}=0)$, el valor de IM es el valor máximo permitido por la ley, que son 35 días. La función de sostenibilidad queda:

$$
\begin{aligned}
& \text { SPM10SS }=e^{-9,9035 \cdot\left(\frac{l}{35}\right)^{2}} \\
& \text { SPM10sl }=e^{-5,3 \cdot\left(\frac{l}{35}\right)^{2}}
\end{aligned}
$$


Para valores de PM10<35 días. Para valores de PM10>35 días la sostenibilidad es igual a cero.

Donde SPM10ss y SPM10s1 representan la sostenibilidad para la concentración de PM10 sensu stricto y sensu lato respectivamente

\section{Tasa de paro (TP)}

Se mide en porcentaje de la población apuntado al INEM.

Lo ideal es que la tasa de paro sea cero, Io $=0 \%$. Hay que definir $\mathrm{I}_{\mathrm{M}}$. Se considera que, como máximo, puede haber un parado en cada unidad familiar, ya que más de un parado por unidad familiar puede resultar bastante insostenible socialmente. Por lo tanto, el valor de IM será el cociente entre el número de unidades familiares (UF) y la población en edad laboral, es decir:

$\mathrm{I}_{\mathrm{M}}=\mathrm{UF} /$ Población edad laboral

Las unidades familiares se pueden estimar, a falta de mayor información, dividiendo la población total entre 2,9, ya que según los datos del INE hay 2,9 individuos en cada unidad familiar como promedio. La población en edad laboral será la que se encuentra en el intervalo de edad entre 16 y 65 años. El valor de IM queda: $\mathrm{I}_{\mathrm{M}}=$ Población total / (2,9*Población edad laboral)

La función de sostenibilidad queda:

$$
\begin{aligned}
& \text { STPSS }=e^{-9,9035 \cdot\left(\frac{l}{M M}\right)^{2}} \\
& \text { STPSl }=e^{-5,3 \cdot\left(\frac{l}{M M}\right)^{2}}
\end{aligned}
$$

Para valores de $\mathrm{TP}<\mathrm{IM}$. Para valores de TP $>\mathrm{IM}$ la sostenibilidad vale cero.

Donde STPss y STPsl representan la sostenibilidad para la tasa de paro sensu stricto y sensu lato respectivamente.

Evidentemente puede existir mucha variación del número de unidades familiares de una ciudad a otra.

\section{Índice de motorización (IM)}

Se mide en vehículos por cada 100 habitantes. Se propone como indicador el número de habitantes por vehículo (HV), es decir el inverso del valor que da el trabajo analizado. Cuando se habla de VAO se considera que el vehículo debe tener una ocupación mínima de tres personas, por lo que se propone este valor como valor óptimo, es decir IO = $3 \mathrm{hab} / \mathrm{veh}$., por lo que la función de sostenibilidad queda:

$$
\text { SIMSS }=e^{-9,9035 \cdot\left(\frac{l-a}{3}\right)^{2}}
$$

$$
S I M s l=e^{-5,3 \cdot\left(\frac{l-3}{3}\right)^{2}}
$$

Para valores de $\mathrm{IM}<3$ hab/veh. Cuando $\mathrm{IM}=>3$ la sostenibilidad vale 1 . 
Donde SIMss y SIMsl representan la sostenibilidad para los habitantes por vehículo sensu stricto y sensu lato respectivamente

Tal y como está definido este indicador, se considera sólo la rama de la izquierda de la función de sostenibilidad.

\section{Ratio viviendas principales/viviendas no principales (VPVNP)}

Mide la relación entre las viviendas principales y las secundarias. Utiliza las mismas definiciones de vivienda vacía y secundaria que utiliza el INE.

Tal y como está definido el indicador, lo ideal es que el cociente sea lo más alto posible; lo ideal es que las viviendas no principales sean cero. Se puede realizar el inverso, es decir el cociente VNP/VP, de tal modo que ahora el valor de Io es cero.

Hay que definir $\mathrm{I}_{\mathrm{M}}$. Parece lógico pensar que una situación insostenible será cuando en una ciudad el número de viviendas no principales supere al de viviendas principales (VNP $>\mathrm{VP})$. Se considera que la sostenibilidad vale cero cuando $\mathrm{VNP}>=\mathrm{VP}$, por lo que $\mathrm{I}_{\mathrm{M}}=1$. La función de sostenibilidad queda:

SVPVNPSS $=e^{-9,9035 \cdot\left(\frac{l}{M}\right)^{2}}$

SVPVNPSl $=e^{-5,3 \cdot\left(\frac{l}{I M}\right)^{2}}$

Cuando VNP/VP=>1 la sostenibilidad vale cero.

SVPVNPss y SVPVNPsl representan la sostenibilidad para el cociente vivienda no principales/viviendas principales sensu stricto y sensu lato respectivamente.

Este indicador puede ser un reflejo del abandono de un núcleo urbano por parte de sus habitantes cuando éstos emigran. También puede ser un reflejo de un exceso de "fiebre constructiva" mal planificada, sobre todo en zonas turísticas. La sostenibilidad vale cero cuando

\section{Densidad urbana (DU)}

Se mide en habitantes por hectárea.

Tal y como está definido el indicador, no da mucha información sobre la sostenibilidad de una ciudad. No está definido cuál es el valor óptimo de densidad urbana que produce un máximo de sostenibilidad.

No existen unos criterios objetivos que permitan definir el valor óptimo de la densidad urbana, ya sean viviendas o habitantes, que produce un máximo de sostenibilidad. El único acuerdo es que la ciudad debe ser compacta.

De los valores de densidad de viviendas señalados en diferentes documentos se toma como valor más próximo a la ciudad compacta el de 75 viv/Ha, que es el recogido en la Ley de Ordenación Urbanística de Andalucía como valor máximo posible.

Según los datos del Instituto Nacional de Estadística hay 2,9 individuos en cada unidad familiar como promedio y cada unidad familiar tendrá una sola vivienda, por lo que el número máximo de habitantes por hectárea será $75 * 2,9=217,5$. Tenemos que Io $=217,5 \mathrm{hab} / \mathrm{Ha}$. La función de sostenibilidad queda: 
SDUSS $=e^{-9,9035 \cdot\left(\frac{I-217,5}{217,5}\right)^{2}}$

SDUsl $=e^{-5,3 \cdot\left(\frac{I-217,5}{217,5}\right)^{2}}$

Para valores de DU $<217,5$ hab/Ha. Para valores superiores a 217,5 hab/Ha la sostenibilidad vale cero.

SDUss y SDUsl representan la sostenibilidad para la densidad urbana sensu stricto y sensu lato respectivamente.

Tal y como está definido el indicador se considera sólo la rama izquierda de la función de sostenibilidad.

Otros indicadores utilizados en el documento analizado se han descartado para este primer estudio:

-Concentración media anual de NOx: No hay datos de muchas ciudades por lo que se perdería capacidad de comparación.

-Índice de actividad económica: Es un indicador que parece un tanto sesgado ya que no recoge las actividades agrícolas ni ganaderas. Las ciudades con amplias superficies agrícolas a su alrededor van a presentar valores más pequeños que las ciudades con menos territorio agrícola.

\section{RESULTADOS}

Los resultados que se obtienen cuando se aplican las distintas expresiones matemáticas a los valores de los indicadores en las diferentes ciudades se muestran en las siguientes tablas, donde se recogen el nombre de la ciudad, las iniciales de los diferentes indicadores, el valor que alcanza cada indicador en cada ciudad (fila I), los valores considerados óptimo y máximo de cada indicador y en cada ciudad (filas Io e IM), y los valores de sostenibilidad sensu stricto y sensu lato para cada indicador (filas Sost ss y Sost sl) y el valor de sostenibilidad media (SM, cuadros amarillo o verde).

\begin{tabular}{|c|c|c|c|c|c|c|c|c|c|c|}
\hline ALBACETE & GRU & CA & CEHe & ZV & PM10 & TP & IM & VNPVP & DU & SM \\
\hline I & 1,16 & 146,6 & 30,45 & 7 & 46 & 7,85 & 1,72 & 0,38 & 57,65 & \\
\hline Io & 0,47 & 37,72 & 9,56 & 20 & 0 & 0 & 3 & 0 & 217,5 & \\
\hline IM & & & & & 35 & 48,96 & & 1 & & \\
\hline Sost ss & 0,0000 & 0,0000 & 0,0000 & 0,0152 & 0,0000 & 0.7752 & 0,1648 & 0,2393 & 0,0048 & 0,1333 \\
\hline Sost sl & 0,00 & 0,00 & 0,00 & 0,11 & 0,00 & 0,87 & 0,38 & 0,47 & 0,06 & 0,21 \\
\hline
\end{tabular}

\begin{tabular}{|c|c|c|c|c|c|c|c|c|c|c|}
\hline ALICANTE & GRU & CA & CEHe & ZV & PM10 & TP & IM & VNPVP & DU & SM \\
\hline I & 1,29 & 139,3 & 19,4 & 10,4 & 37 & 6,76 & 1,64 & 0,49 & 67,59 & \\
\hline Io & 0,47 & 31,96 & 9,5 & 20 & 0 & 0 & 3 & 0 & 217,5 & \\
\hline IM & & & & & 35 & 49,64 & & 1 & & \\
\hline Sost ss & 0,0000 & 0,0000 & 0,0000 & 0,1021 & 0,0000 & 0.8322 & 0,1306 & 0,0928 & 0,0091 & 0,1296 \\
\hline Sost sl & 0,00 & 0,00 & 0,00 & 0,29 & 0,00 & 0,91 & 0,34 & 0,28 & 0,08 & 0,21 \\
\hline
\end{tabular}




\begin{tabular}{|}
\begin{tabular}{|c|c|c|c|c|c|c|c|c|c|c|}
\hline ALMERIA & GRU & CA & CEHe & ZV & PM10 & TP & IM & VNPVP & DU & SM \\
\hline I & 0,8 & 105,9 & 17,89 & - & 43 & 7,83 & 1,67 & 0,46 & 73,16 & \\
\hline Io & 0,46 & 22,28 & 9,52 & 20 & 0 & 0 & 3 & 0 & 217,5 & \\
\hline IM & & & & & 35 & 49,24 & & 1 & & \\
\hline Sost ss & 0,0045 & 0,0000 & 0,0005 & - & 0,0000 & 0,7785 & 0,1428 & 0,1230 & 0,0128 & 0,1327 \\
\hline Sost sl & 0,06 & 0,00 & 0,02 & - & 0,00 & 0,87 & 0,35 & 0,33 & 0,10 & 0,22 \\
\hline ÁVILA & GRU & CA & CEHe & ZV & PM10 & TP & IM & VNPVP & DU & SM \\
\hline I & 1,09 & - & 29,83 & 7 & 26 & 7,54 & 1,65 & 0,5 & 36,01 & \\
\hline Io & 0,47 & 43,18 & 9,53 & 20 & 0 & 0 & 3 & 0 & 217,5 & \\
\hline IM & & & & & 35 & 49,54 & & 1 & & \\
\hline Sost ss & 0,0000 & - & 0,0000 & 0,0152 & 0,0042 & 0,7950 & 0,1346 & 0,0841 & 0,0010 & 0,1293 \\
\hline Sost sl & 0,00 & - & 0,00 & 0,11 & 0,05 & 0,88 & 0,34 & 0,27 & 0,02 & 0,21 \\
\hline
\end{tabular}
\end{tabular}

\begin{tabular}{|c|c|c|c|c|c|c|c|c|c|c|}
\hline BADAJOZ & GRU & CA & CEHe & ZV & PM10 & TP & IM & VNPVP & DU & SM \\
\hline I & 0,97 & 172,3 & 22,12 & - & 5 & 10,47 & 1,59 & 0,38 & 23,67 & \\
\hline Io & 0,46 & 48,47 & 9,51 & 20 & 0 & 0 & 3 & 0 & 217,5 & \\
\hline IM & & & & & 35 & 48,99 & & 1 & & \\
\hline Sost ss & 0,0000 & 0,0000 & 0,0000 & - & 0,8170 & 0,6361 & 0,1122 & 0,2393 & 0,0004 & 0,2256 \\
\hline Sost s1 & 0,00 & 0,00 & 0,00 & - & 0,90 & 0,78 & 0,31 & 0,47 & 0,01 & 0,31 \\
\hline
\end{tabular}

\begin{tabular}{|c|c|c|c|c|c|c|c|c|c|c|}
\hline BARCELONA & GRU & CA & CEHe & ZV & PM10 & TP & IM & VNPVP & DU & SM \\
\hline I & 1,47 & 121,6 & 26,59 & 6,6 & 44 & 4,88 & 1,75 & 0,27 & 199,7 & \\
\hline Io & 0,48 & 71,45 & 9,47 & 20 & 0 & 0 & 3 & 0 & 217,5 & \\
\hline IM & & & & & 35 & 50,86 & & 1 & & \\
\hline Sost ss & 0,0000 & 0,0000 & 0,0000 & 0,117 & 0,0000 & 0,9129 & 0,1792 & 0,4858 & 0,9356 & 0,2814 \\
\hline Sost s1 & 0,00 & 0,07 & 0,00 & 0,09 & 0,00 & 095 & 0,40 & 0,68 & 0,97 & 0,35 \\
\hline
\end{tabular}

\begin{tabular}{|c|c|c|c|c|c|c|c|c|c|c|}
\hline BILBAO & GRU & CA & CEHe & ZV & PM10 & TP & IM & VNPVP & DU & SM \\
\hline I & 1,26 & 112,6 & 50,47 & 6,1 & 56 & 6,29 & 2,02 & 0,13 & 212,5 & \\
\hline Io & 0,48 & 141,95 & 9,44 & 20 & 0 & 0 & 3 & 0 & 217,5 & \\
\hline IM & & & & & 35 & 51,55 & & 1 & & \\
\hline Sost ss & 0,0000 & 1,0000 & 0,0000 & 0,0084 & 0,0000 & 0,8629 & 0,3476 & 0,8459 & 0,9947 & 0,4510 \\
\hline Sost sl & 0,00 & 1,00 & 0,00 & 0,08 & 0,00 & 0,92 & 0,57 & 0,91 & 1,00 & 0,50 \\
\hline
\end{tabular}

\begin{tabular}{|c|c|c|c|c|c|c|c|c|c|c|}
\hline BURGOS & GRU & CA & CEHe & ZV & PM10 & TP & IM & VNPVP & DU & SM \\
\hline I & 10,7 & 117,5 & 42,6 & - & 23 & 5,93 & 1,87 & 0,31 & 44,17 & \\
\hline Io & 0,47 & 62,57 & 9,48 & 20 & 0 & 0 & 3 & 0 & 217,5 & \\
\hline IM & & & & & 35 & 49,82 & & 1 & & \\
\hline Sost ss & 0,0000 & 0,0005 & 0,0000 & - & 0,0139 & 0,8691 & 0,2453 & 0,3861 & 0,0019 & 0,1896 \\
\hline Sost sl & 0,00 & 0,02 & 0,00 & - & 0,10 & 0,93 & 0,47 & 0,60 & 0,03 & 0,27 \\
\hline
\end{tabular}

\begin{tabular}{|c|c|c|c|c|c|c|c|c|c|c|}
\hline CÁCERES & GRU & CA & CEHe & ZV & PM10 & TP & IM & VNPVP & DU & SM \\
\hline I & 1,19 & 145,3 & 19,63 & 16,6 & - & 8,27 & 1,6 & 0,5 & 35,79 & \\
\hline Io & 0,47 & 56,78 & 9,51 & 20 & 0 & 0 & 3 & 0 & 217,5 & \\
\hline IM & & & & & 35 & 48,61 & & 1 & & \\
\hline Sost ss & 0,0000 & 0,0000 & 0,0000 & 0,7511 & - & 0,7508 & 0,1157 & 0,0841 & 0,0010 & 0,2128 \\
\hline Sost sl & 0,00 & 0,00 & 0,00 & 0,86 & - & 0,86 & 0,32 & 0,27 & 0,02 & 0,29 \\
\hline
\end{tabular}




\begin{tabular}{|c|c|c|c|c|c|c|c|c|c|c|}
\hline CÁDIZ & GRU & CA & CEHe & ZV & PM10 & TP & IM & VNPVP & DU & SM \\
\hline I & 1,32 & 201,9 & 30,22 & 17,6 & 18 & 12,9 & 2,12 & 0,25 & 178,2 & \\
\hline Io & 0,48 & 64,97 & 9,48 & 20 & 0 & 0 & 3 & 0 & 217,5 & \\
\hline IM & & & & & 35 & 49,24 & & 1 & & \\
\hline Sost ss & 0,0000 & 0,0000 & 0,0000 & 0,8671 & 0,0728 & 0,5068 & 0,4265 & 0,5385 & 0,7231 & 0,3483 \\
\hline Sost sl & 0,00 & 0,00 & 0,00 & 0,93 & 0,25 & 0,70 & 0,63 & 0,72 & 0,84 & 0,45 \\
\hline
\end{tabular}

\begin{tabular}{|c|c|c|c|c|c|c|c|c|c|c|}
\hline CASTELLÓN & GRU & CA & CEHe & ZV & PM10 & TP & IM & VNPVP & DU & SM \\
\hline I & 1,18 & - & 75,85 & 7,8 & 26 & 4,1 & 1,5 & 0,27 & 81,13 & \\
\hline Io & 0,47 & 45,75 & 9,55 & 20 & 0 & 0 & 3 & 0 & 217,5 & \\
\hline IM & & & & & 35 & 48,51 & & 1 & & \\
\hline Sost ss & 0,0000 & - & 0,0000 & 0,0251 & 0,0042 & 0,9317 & 0,0841 & 0,4858 & 0,0204 & 0,1939 \\
\hline Sost sl & 0,00 & - & 0,00 & 0,14 & 0,05 & 0,96 & 0,27 & 0,68 & 0,12 & 0,28 \\
\hline
\end{tabular}

\begin{tabular}{|c|c|c|c|c|c|c|c|c|c|c|}
\hline CIUDAD REAL & GRU & CA & CEHe & ZV & PM10 & TP & IM & VNPVP & DU & SM \\
\hline I & 1,3 & - & 34,48 & - & - & 8,04 & 1,83 & 0,38 & 36,29 & \\
\hline Io & 0,46 & 40,89 & 9,49 & 20 & 0 & 0 & 3 & 0 & 217,5 & \\
\hline IM & & & & & 35 & 49,33 & & 1 & & \\
\hline Sost ss & 0,0000 & - & 0,0000 & - & - & 0,7687 & 0,2217 & 0,2393 & 0,0010 & 0,2051 \\
\hline Sost sl & 0,00 & - & 0,00 & - & - & 0,87 & 0,45 & 0,47 & 0,03 & 0,30 \\
\hline
\end{tabular}

\begin{tabular}{|c|c|c|c|c|c|c|c|c|c|c|}
\hline CÓRDOBA & GRU & CA & CEHe & ZV & PM10 & TP & IM & VNPVP & DU & SM \\
\hline I & 1,2 & 155,7 & 17,89 & 4,6 & 19 & 12,1 & 1,82 & 0,3 & 53,18 & \\
\hline Io & 0,47 & 56,98 & 9,46 & 20 & 0 & 0 & 3 & 0 & 217,5 & \\
\hline IM & & & & & 35 & 50,13 & & 1 & & \\
\hline Sost ss & 0,0000 & 0,0000 & 0,0004 & 0,0028 & 0,0540 & 0,5616 & 0,2161 & 0,4101 & 0,0035 & 0,1387 \\
\hline Sost sl & 0,00 & 0,00 & 0,01 & 0,04 & 0,21 & 0,73 & 0,44 & 0,62 & 0,05 & 0,23 \\
\hline
\end{tabular}

\begin{tabular}{|c|c|c|c|c|c|c|c|c|c|c|}
\hline CORUÑA (LA) & GRU & CA & CEHe & ZV & PM10 & TP & IM & VNPVP & DU & SM \\
\hline I & 1,2 & 112,3 & 23,55 & 8,2 & 23 & 8,37 & 1,72 & 0,35 & 94,62 & \\
\hline Io & 0,48 & 118,4 & 9,44 & 20 & 0 & 0 & 3 & 0 & 217,5 & \\
\hline IM & & & & & 35 & 50,11 & & 1 & & \\
\hline Sost ss & 0,0000 & 1,0000 & 0,0000 & 0,0318 & 0,0139 & 0,7586 & 0,1648 & 0,2973 & 0,0424 & 0,2565 \\
\hline Sost sl & 0,00 & 1,00 & 0,00 & 0,16 & 0,10 & 0,86 & 0,38 & 0,52 & 0,18 & 0,36 \\
\hline
\end{tabular}

\begin{tabular}{|c|c|c|c|c|c|c|c|c|c|c|}
\hline CUENCA & GRU & CA & CEHe & ZV & PM10 & TP & IM & VNPVP & DU & SM \\
\hline I & 1,3 & - & 30,62 & 8,7 & 56 & 6,48 & 1,68 & 0,51 & 47,81 & \\
\hline Io & 0,47 & 55,86 & 9,55 & 20 & 0 & 0 & 3 & 0 & 217,5 & \\
\hline IM & & & & & 35 & 50,01 & & 1 & & \\
\hline Sost ss & 0,0000 & - & 0,0000 & 0,0424 & 0,0000 & 0,8468 & 0,1470 & 0,0761 & 0,0024 & 0,1393 \\
\hline Sost sl & 0,00 & - & 0,00 & 0,18 & 0,00 & 0,91 & 0,36 & 0,25 & 0,04 & 0,22 \\
\hline
\end{tabular}

\begin{tabular}{|c|c|c|c|c|c|c|c|c|c|c|}
\hline GERONA & GRU & CA & CEHe & ZV & PM10 & TP & IM & VNPVP & DU & SM \\
\hline I & 1,47 & 170,1 & 25,52 & 24,3 & 13 & 4,68 & 1,48 & 0,48 & 64,36 & \\
\hline Io & 0,47 & 85,52 & 9,53 & 20 & 0 & 0 & 3 & 0 & 217,5 & \\
\hline IM & & & & & 35 & 48,65 & & 1 & & \\
\hline Sost ss & 0,0000 & 0,0000 & 0,0000 & 1,0000 & 0,2551 & 0,9124 & 0,0787 & 0,1021 & 0,0074 & 0,2617 \\
\hline Sost sl & 0,00 & 0,00 & 0,00 & 1,00 & 0,48 & 0,95 & 0,26 & 0,29 & 0,07 & 0,34 \\
\hline
\end{tabular}




\begin{tabular}{|c|c|c|c|c|c|c|c|c|c|c|}
\hline GRANADA & GRU & CA & CEHe & ZV & PM10 & TP & IM & VNPVP & DU & SM \\
\hline I & 1,5 & 140,8 & 20,15 & - & 44 & 9,63 & 1,5 & 0,53 & 88,59 & \\
\hline Io & 0,48 & 35,85 & 9,48 & 20 & 0 & 0 & 3 & 0 & 217,5 & \\
\hline IM & & & & & 35 & 49,76 & & 1 & & \\
\hline Sost ss & 0,0000 & 0,0000 & 0,0000 & - & 0,0000 & 0,6901 & 0,0841 & 0,0619 & 0,0308 & 0,1084 \\
\hline Sost sl & 0,00 & 0,00 & 0,00 & - & 0,00 & 0,82 & 0,27 & 0,23 & 0,16 & 0,18 \\
\hline
\end{tabular}

\begin{tabular}{|c|c|c|c|c|c|c|c|c|c|c|}
\hline GUADALAJARA & GRU & CA & CEHe & ZV & PM10 & TP & IM & VNPVP & DU & SM \\
\hline I & 1,5 & 170,3 & 40,1 & - & 14 & 4,7 & 1,62 & 0,28 & 41,23 & \\
\hline Io & 0,47 & 55,85 & 9,56 & 20 & 0 & 0 & 3 & 0 & 217,5 & \\
\hline IM & & & & & 35 & 48,58 & & 1 & & \\
\hline Sost ss & 0,0000 & 0,0000 & 0,0000 & - & 0,2050 & 0,9115 & 0,1230 & 0,4600 & 0,0015 & 0,2126 \\
\hline Sost sl & 0,00 & 0,00 & 0,00 & - & 0,43 & 0,95 & 0,33 & 0,66 & 0,03 & 030 \\
\hline
\end{tabular}

\begin{tabular}{|c|c|c|c|c|c|c|c|c|c|c|}
\hline HUELVA & GRU & CA & CEHe & ZV & PM10 & TP & IM & VNPVP & DU & SM \\
\hline I & 1,33 & 164,6 & 67,57 & 6 & 13 & 9,63 & 1,76 & 0,33 & 78,36 & \\
\hline Io & 0,47 & 51,19 & 9,5 & 20 & 0 & 0 & 3 & 0 & 217,5 & \\
\hline IM & & & & & 35 & 48,94 & & 1 & & \\
\hline Sost ss & 0,0000 & 0,0000 & 0,0000 & 0,0078 & 0,2551 & 0,6815 & 0,1842 & 0,3401 & 0,0174 & 0,1651 \\
\hline Sost sl & 0,00 & 0,00 & 0,00 & 0,07 & 0,48 & 0,81 & 0,40 & 0,56 & 0,11 & 0,27 \\
\hline
\end{tabular}

\begin{tabular}{|c|c|c|c|c|c|c|c|c|c|c|}
\hline HUESCA & GRU & CA & CEHe & ZV & PM10 & TP & IM & VNPVP & DU & SM \\
\hline I & 1,09 & 148,1 & 45,4 & 10,1 & 5 & 4,65 & 1,61 & 0,43 & 32,62 & \\
\hline Io & 0,47 & 58,94 & 9,47 & 20 & 0 & 0 & 3 & 0 & 217,5 & \\
\hline IM & & & & & 35 & 50,79 & & 1 & & \\
\hline Sost ss & 0,0000 & 0,0000 & 0,0000 & 0,0883 & 0,8170 & 0,9203 & 0,1193 & 0,1602 & 0,0008 & 0,2340 \\
\hline Sost sl & 0,00 & 0,00 & 0,00 & 0,27 & 0,90 & 0,96 & 0,32 & 0,38 & 0,02 & 0,32 \\
\hline
\end{tabular}

\begin{tabular}{|c|c|c|c|c|c|c|c|c|c|c|}
\hline JAÉN & GRU & CA & CEHe & ZV & PM10 & TP & IM & VNPVP & DU & SM \\
\hline I & 0,97 & 161,7 & 20,01 & - & 24 & 8,41 & 1,78 & 0,33 & 59,79 & \\
\hline Io & 0,46 & 66,48 & 9,49 & 20 & 0 & 0 & 3 & 0 & 217,5 & \\
\hline IM & & & & & 35 & 50,12 & & 1 & & \\
\hline Sost ss & 0,0000 & 0,0000 & 0,0000 & - & 0,0095 & 0,7567 & 0,1944 & 0,3401 & 0,0055 & 0,1633 \\
\hline Sost sl & 0,00 & 0,00 & 0,00 & - & 0,08 & 0,86 & 0,42 & 0,56 & 0,03 & 0,25 \\
\hline
\end{tabular}

\begin{tabular}{|c|c|c|c|c|c|c|c|c|c|c|}
\hline LEÓN & GRU & CA & CEHe & ZV & PM10 & TP & IM & VNPVP & DU & SM \\
\hline I & 1,23 & 151,1 & 30,24 & 14,8 & 37 & 7,64 & 1,83 & 0,38 & 64,25 & \\
\hline Io & 0,48 & 62,64 & 9,41 & 20 & 0 & 0 & 3 & 0 & 217,5 & \\
\hline IM & & & & & 35 & 51,79 & & 1 & & \\
\hline Sost ss & 0,0000 & 0,0000 & 0,0000 & 0,5120 & 0,0000 & 0,8061 & 0,2217 & 0,2393 & 0,0073 & 0,1985 \\
\hline Sost sl & 0,00 & 0,00 & 0,00 & 0,70 & 0,00 & 0,89 & 0,45 & 0,47 & 0,07 & 0,29 \\
\hline
\end{tabular}

\begin{tabular}{|c|c|c|c|c|c|c|c|c|c|c|}
\hline LÉRIDA & GRU & CA & CEHe & ZV & PM10 & TP & IM & VNPVP & DU & SM \\
\hline I & 1,17 & 166,6 & 30,66 & 9,4 & 17 & 4,35 & 1,67 & 0,42 & 57,37 & \\
\hline Io & 0,47 & 37,49 & 9,54 & 20 & 0 & 0 & 3 & 0 & 217,5 & \\
\hline IM & & & & & 35 & 49,53 & & 1 & & \\
\hline Sost ss & 0,0000 & 0,0000 & 0,0000 & 0,0619 & 0,0967 & 0,9265 & 0,1428 & 0,1743 & 0,0047 & 0,1563 \\
\hline Sost sl & 0,00 & 0,00 & 0,00 & 0,23 & 0,29 & 0,96 & 0,35 & 0,39 & 0,06 & 0,25 \\
\hline
\end{tabular}




\begin{tabular}{|c|c|c|c|c|c|c|c|c|c|c|}
\hline LOGROÑO & GRU & CA & CEHe & ZV & PM10 & TP & IM & VNPVP & DU & SM \\
\hline I & 0,95 & 130,9 & 53,63 & 19,9 & 5 & 5,38 & 1,98 & 0,31 & 88,43 & \\
\hline Io & 0,47 & 41,87 & 9,48 & 20 & 0 & 0 & 3 & 0 & 217,5 & \\
\hline IM & & & & & 35 & 49,66 & & 1 & & \\
\hline Sost ss & 0,0000 & 0,0000 & 0,0000 & 0,9998 & 0,8170 & 0,8903 & 0,3183 & 0,3861 & 0,0306 & 0,3824 \\
\hline Sost sl & 0,00 & 0,00 & 0,00 & 1,00 & 0,90 & 0,94 & 0,54 & 0,60 & 0,15 & 0,46 \\
\hline
\end{tabular}

\begin{tabular}{|c|c|c|c|c|c|c|c|c|c|c|}
\hline LUGO & GRU & CA & CEHe & ZV & PM10 & TP & IM & VNPVP & DU & SM \\
\hline I & 1,16 & 132,7 & 32,9 & 11,8 & 7 & 8,52 & 1,6 & 0,61 & 82,61 & \\
\hline Io & 0,48 & 128,89 & 9,45 & 20 & 0 & 0 & 3 & 0 & 217,5 & \\
\hline IM & & & & & 35 & 50,31 & & 1 & & \\
\hline Sost ss & 0,0000 & 0,9914 & 0,0000 & 0,1892 & 0,6729 & 0,7527 & 0,1157 & 0,0251 & 0,0222 & 0,3077 \\
\hline Sost sl & 0,00 & 1,00 & 0,00 & 0,41 & 0,81 & 0,86 & 0,32 & 0,14 & 0,13 & 0,41 \\
\hline
\end{tabular}

\begin{tabular}{|c|c|c|c|c|c|c|c|c|c|c|}
\hline MADRID & GRU & CA & CEHe & ZV & PM10 & TP & IM & VNPVP & DU & SM \\
\hline I & 1,33 & 157,9 & 19,72 & 17 & 24 & 5,14 & 1,61 & 0,28 & 86,91 & \\
\hline Io & 0,48 & 46,02 & 9,47 & 20 & 0 & 0 & 3 & 0 & 217,5 & \\
\hline IM & & & & & 35 & 50,35 & & 1 & & \\
\hline Sost ss & 0,0000 & 0,0000 & 0,0000 & 0,8003 & 0,0095 & 0,9019 & 0,1193 & 0,4600 & 0,0282 & 0,2577 \\
\hline Sost sl & 0,00 & 0,00 & 0,00 & 0,89 & 0,08 & 0,95 & 0,32 & 0,66 & 0,15 & 0,34 \\
\hline
\end{tabular}

\begin{tabular}{|c|c|c|c|c|c|c|c|c|c|c|}
\hline MÁLAGA & GRU & CA & CEHe & ZV & PM10 & TP & IM & VNPVP & DU & SM \\
\hline I & 1,63 & 133,3 & 17,83 & 5,7 & 18 & 9,96 & 1,64 & 0,27 & 73,3 & \\
\hline Io & 0,47 & 55,58 & 9,49 & 20 & 0 & 0 & 3 & 0 & 217,5 & \\
\hline IM & & & & & 35 & 49,12 & & 1 & & \\
\hline Sost ss & 0,0000 & 0,0000 & 0,0005 & 0,0063 & 0,0728 & 0,6655 & 0,1306 & 0,4858 & 0,0129 & 0,1527 \\
\hline Sost sl & 0,00 & 0,00 & 0,02 & 0,07 & 0,25 & 0,80 & 0,34 & 0,68 & 0,10 & 0,25 \\
\hline
\end{tabular}

\begin{tabular}{|c|c|c|c|c|c|c|c|c|c|c|}
\hline MURCIA & GRU & CA & CEHe & ZV & PM10 & TP & IM & VNPVP & DU & SM \\
\hline I & 1,26 & 155,2 & 39,25 & 4,2 & 7 & 4,25 & 1,55 & 0,29 & 36,54 & \\
\hline Io & 0,46 & 27,41 & 9,53 & 20 & 0 & 0 & 3 & 0 & 217,5 & \\
\hline IM & & & & & 35 & 49,43 & & 1 & & \\
\hline Sost ss & 0,0000 & 0,0000 & 0,0000 & 0,0021 & 0,6729 & 0,9294 & 0,0989 & 0,4348 & 0,0011 & 0,2377 \\
\hline Sost sl & 0,00 & 0,00 & 0,00 & 0,04 & 0,81 & 0,96 & 0,29 & 0,64 & 0,03 & 0,31 \\
\hline
\end{tabular}

\begin{tabular}{|c|c|c|c|c|c|c|c|c|c|c|}
\hline ORENSE & GRU & CA & CEHe & ZV & PM10 & TP & IM & VNPVP & DU & SM \\
\hline I & 1,08 & 137,5 & 22,11 & 8,2 & 9 & 9,76 & 1,43 & 0,5 & 43,54 & \\
\hline Io & 0,48 & 94,19 & 9,43 & 20 & 0 & 0 & 3 & 0 & 217,5 & \\
\hline IM & & & & & 35 & 51,31 & & 1 & & \\
\hline Sost ss & 0,0000 & 0,1232 & 0,0000 & 0,0318 & 0,5195 & 0,6988 & 0,0664 & 0,0841 & 0,0018 & 0,1695 \\
\hline Sost sl & 0,00 & 0,33 & 0,00 & 0,16 & 0,70 & 0,83 & 0,23 & 0,27 & 0,03 & 0,28 \\
\hline
\end{tabular}

\begin{tabular}{|c|c|c|c|c|c|c|c|c|c|c|}
\hline OVIEDO & GRU & CA & CEHe & ZV & PM10 & TP & IM & VNPVP & DU & SM \\
\hline I & 1,05 & - & 26,12 & - & 24 & 6,94 & 1,97 & 0,32 & 123,3 & \\
\hline Io & 0,48 & 114,5 & 9,46 & 20 & 0 & 0 & 3 & 0 & 217,5 & \\
\hline IM & & & & & 35 & 48,85 & & 1 & & \\
\hline Sost ss & 0,0000 & - & 0,0000 & - & 0,0095 & 0,8188 & 0,3112 & 0,3627 & 0,1558 & 0,2369 \\
\hline Sost sl & 0,00 & - & 0,00 & - & 0,08 & 0,90 & 0,54 & 0,58 & 0,37 & 0,35 \\
\hline
\end{tabular}




\begin{tabular}{|c|c|c|c|c|c|c|c|c|c|c|}
\hline PALENCIA & GRU & CA & CEHe & ZV & PM10 & TP & IM & VNPVP & DU & SM \\
\hline I & 1,05 & 167,2 & 34,33 & 10 & 34 & 8,11 & 1,89 & 0,34 & 70,56 & \\
\hline Io & 0,48 & 44,43 & 9,46 & 20 & 0 & 0 & 3 & 0 & 217,5 & \\
\hline IM & & & & & 35 & 50,07 & & 1 & & \\
\hline Sost ss & 0,0000 & 0,0000 & 0,0000 & 0,0841 & 0,0001 & 0,7712 & 0,2577 & 0,3183 & 0,0109 & 0,1603 \\
\hline Sost sl & 0,00 & 0,00 & 0,00 & 0,27 & 0,01 & 0,87 & 0,48 & 0,54 & 0,09 & 0,25 \\
\hline
\end{tabular}

\begin{tabular}{|c|c|c|c|c|c|c|c|c|c|c|}
\hline PALMA MALLO. & GRU & CA & CEHe & ZV & PM10 & TP & IM & VNPVP & DU & SM \\
\hline I & 1,75 & 140,4 & 29,13 & - & 9 & 5,65 & 1,29 & 0,31 & 66,05 & \\
\hline Io & 0,47 & 43,33 & 9,56 & 20 & 0 & 0 & 3 & 0 & 217,5 & \\
\hline IM & & & & & 35 & 47,77 & & 1 & & \\
\hline Sost ss & 0,0000 & 0,0000 & 0,0000 & - & 0,5195 & 0,8706 & 0,0400 & 0,3861 & 0,0082 & 0,2281 \\
\hline Sost sl & 0,00 & 0,00 & 0,00 & - & 0,70 & 0,93 & 0,18 & 0,60 & 0,08 & 0,31 \\
\hline
\end{tabular}

\begin{tabular}{|c|c|c|c|c|c|c|c|c|c|c|}
\hline Las PALMAS & GRU & CA & CEHe & ZV & PM10 & TP & IM & VNPVP & DU & SM \\
\hline I & 1,2 & - & 14,53 & 1,6 & 14 & 11,37 & 1,72 & 0,34 & 116,3 & \\
\hline Io & 0,48 & 4,87 & 9,46 & 20 & 0 & 0 & 3 & 0 & 217,5 & \\
\hline IM & & & & & 35 & 50,07 & & 1 & & \\
\hline Sost ss & 0,0000 & - & 0,0582 & 0,0002 & 0,2050 & 0,6001 & 0,1648 & 0,3183 & 0,1174 & 0,1830 \\
\hline Sost sl & 0,00 & - & 0,22 & 0,01 & 0,43 & 0,76 & 0,38 & 0,54 & 0,32 & 0,33 \\
\hline
\end{tabular}

\begin{tabular}{|c|c|c|c|c|c|c|c|c|c|c|}
\hline PAMPLONA & GRU & CA & CEHe & ZV & PM10 & TP & IM & VNPVP & DU & SM \\
\hline I & 1.24 & 145,3 & 42,91 & 0,8 & 17 & 6,06 & 1,67 & 0,31 & 102,4 & \\
\hline Io & 0,47 & 82,85 & 9,45 & 20 & 0 & 0 & 3 & 0 & 217,5 & \\
\hline IM & & & & & 35 & 50,62 & & 1 & & \\
\hline Sost ss & 0,0000 & 0,0036 & 0,0000 & 0,0001 & 0,0967 & 0,8677 & 0,1428 & 0,3861 & 0,0626 & 0,1733 \\
\hline Sost sl & 0,00 & 0,05 & 0,00 & 0,01 & 0,29 & 0,93 & 0,35 & 0,60 & 0,23 & 0,27 \\
\hline
\end{tabular}

\begin{tabular}{|c|c|c|c|c|c|c|c|c|c|c|}
\hline PONTEVEDRA & GRU & CA & CEHe & ZV & PM10 & TP & IM & VNPVP & DU & SM \\
\hline I & - & 162,3 & 18,87 & - & 0 & 8,63 & 1,56 & 0,39 & 60,76 & \\
\hline Io & 0,47 & 204,25 & 9,47 & 20 & 0 & 0 & 3 & 0 & 217,5 & \\
\hline IM & & & & & 35 & 49,29 & & 1 & & \\
\hline Sost ss & - & 1,0000 & 0,0001 & - & 1,0000 & 0,7382 & 0,1021 & 0,2217 & 0,0058 & 0,4383 \\
\hline Sost sl & - & 1,00 & 0,01 & - & 1,00 & 0,85 & 0,29 & 0,45 & 0,06 & 0,52 \\
\hline
\end{tabular}

\begin{tabular}{|c|c|c|c|c|c|c|c|c|c|c|}
\hline SALAMANCA & GRU & CA & CEHe & ZV & PM10 & TP & IM & VNPVP & DU & SM \\
\hline I & 1,2 & 172,7 & 27,83 & - & 36 & 8,94 & 1,96 & 0,43 & 59,04 & \\
\hline Io & 0,48 & 40,40 & 9,41 & 20 & 0 & 0 & 3 & 0 & 217,5 & \\
\hline IM & & & & & 35 & 52,01 & & 1 & & \\
\hline Sost ss & 0,0000 & 0,0000 & 0,0000 & - & 0,0000 & 0,7463 & 0,3042 & 0,1602 & 0,0052 & 0,1520 \\
\hline Sost sl & 0,00 & 0,00 & 0,00 & - & 0,00 & 0,86 & 0,53 & 0,38 & 0,06 & 0,23 \\
\hline
\end{tabular}

\begin{tabular}{|c|c|c|c|c|c|c|c|c|c|c|}
\hline S. SEBASTIÁN & GRU & CA & CEHe & ZV & PM10 & TP & IM & VNPVP & DU & SM \\
\hline I & 1,34 & 163,5 & 41,63 & 22,5 & 3 & 5,01 & 1,77 & 0,4 & 93,69 & \\
\hline Io & 0,48 & 188,96 & 9,41 & 20 & 0 & 0 & 3 & 0 & 217,5 & \\
\hline IM & & & & & 35 & 51,2 & & 1 & & \\
\hline Sost ss & 0,0000 & 1,0000 & 0,0000 & 1,0000 & 0,9298 & 0,9095 & 0,1892 & 0,2020 & 0,0404 & 0,4749 \\
\hline Sost sl & 0,00 & 1,00 & 0,00 & 1,00 & 0,96 & 0,95 & 0,41 & 0,43 & 0,18 & 0,55 \\
\hline
\end{tabular}




\begin{tabular}{|c|c|c|c|c|c|c|c|c|c|c|}
\hline SANTA CRUZ & GRU & CA & CEHe & ZV & PM10 & TP & IM & VNPVP & DU & SM \\
\hline I & 1,35 & 120,3 & 15,77 & 5,5 & 30 & 8,2 & 1,42 & 0,32 & 77,69 & \\
\hline Io & 0,48 & 14,53 & 9,56 & 20 & 0 & 0 & 3 & 0 & 217,5 & \\
\hline IM & & & & & 35 & 47,95 & & 1 & & \\
\hline Sost ss & 0,0000 & 0,0000 & 0,0153 & 0,0055 & 0,0007 & 0,7485 & 0,0641 & 0,3627 & 0,0167 & 0,1348 \\
\hline Sost sl & 0,00 & 0,00 & 0,11 & 0,06 & 0,02 & 0,86 & 0,23 & 0,58 & 0,11 & 0,22 \\
\hline
\end{tabular}

\begin{tabular}{|c|c|c|c|c|c|c|c|c|c|c|}
\hline SANTANDER & GRU & CA & CEHe & ZV & PM10 & TP & IM & VNPVP & DU & SM \\
\hline I & 1,6 & 187 & 32,16 & 7,9 & 45 & 5,98 & 1,76 & 0,3 & 74,87 & \\
\hline Io & 0,48 & 148,43 & 9,45 & 20 & 0 & 0 & 3 & 0 & 217,5 & \\
\hline IM & & & & & 35 & 50,11 & & 1 & & \\
\hline Sost ss & 0,0000 & 0,5124 & 0,0000 & 0,0267 & 0,0000 & 0,8685 & 0,1842 & 0,4101 & 0,0141 & 0,2240 \\
\hline Sost sl & 0,00 & 0,70 & 0,00 & 0,14 & 0,00 & 0,93 & 0,40 & 0,62 & 0,10 & 0,32 \\
\hline
\end{tabular}

\begin{tabular}{|c|c|c|c|c|c|c|c|c|c|c|}
\hline SEGOVIA & GRU & CA & CEHe & ZV & PM10 & TP & IM & VNPVP & DU & SM \\
\hline I & 1,3 & 151,5 & 37,59 & 11,4 & 21 & 4,33 & 1,61 & 0,39 & 54,99 & \\
\hline Io & 0,47 & 50,68 & 9,5 & 20 & 0 & 0 & 3 & 0 & 217,5 & \\
\hline IM & & & & & 35 & 51,25 & & 1 & & \\
\hline Sost ss & 0,0000 & 0,0000 & 0,0000 & 0,1602 & 0,0283 & 0,9317 & 0,1193 & 0,2217 & 0,0040 & 0,1628 \\
\hline Sost sl & 0,00 & 0,00 & 0,00 & 0,38 & 0,15 & 0,96 & 0,32 & 0,45 & 0,05 & 0,26 \\
\hline
\end{tabular}

\begin{tabular}{|c|c|c|c|c|c|c|c|c|c|c|}
\hline SEVILLA & GRU & CA & CEHe & ZV & PM10 & TP & IM & VNPVP & DU & SM \\
\hline I & 1,41 & 137 & 18,48 & 6,2 & 59 & 9,38 & 1,69 & 0,31 & 71,78 & \\
\hline Io & 0,47 & 56.22 & 9,47 & 20 & 0 & 0 & 3 & 0 & 217,5 & \\
\hline IM & & & & & 35 & 49,81 & & 1 & & \\
\hline Sost ss & 0,0000 & 0,0000 & 0,0001 & 0,0090 & 0,0000 & 0,7038 & 0,1513 & 0,3861 & 0,0117 & 0,1402 \\
\hline Sost sl & 0,00 & 0,00 & 0,01 & 0,08 & 0,00 & 0,83 & 0,36 & 0,60 & 0,09 & 0,22 \\
\hline
\end{tabular}

\begin{tabular}{|c|c|c|c|c|c|c|c|c|c|c|}
\hline SORIA & GRU & CA & CEHe & ZV & PM10 & TP & IM & VNPVP & DU & SM \\
\hline I & 1 & 161,9 & 47,23 & 12,8 & 45 & 5,27 & 1,52 & 0,57 & 58,68 & \\
\hline Io & 0,47 & 55,92 & 9,45 & 20 & 0 & 0 & 3 & 0 & 217,5 & \\
\hline IM & & & & & 35 & 51,93 & & 1 & & \\
\hline Sost ss & 0,0000 & 0,0000 & 0,0000 & 0,2771 & 0,0000 & 0,9030 & 0,0898 & 0,0400 & 0,0051 & 01461 \\
\hline Sost sl & 0,00 & 0,00 & 0,00 & 0,50 & 0,00 & 0,95 & 0,28 & 0,18 & 0,06 & 0,22 \\
\hline
\end{tabular}

\begin{tabular}{|c|c|c|c|c|c|c|c|c|c|c|}
\hline TARRAGONA & GRU & CA & CEHe & ZV & PM10 & TP & IM & VNPVP & DU & SM \\
\hline I & 1,4 & 217 & 51,97 & - & 19 & 5,38 & 1,6 & 0,43 & 44,9 & \\
\hline Io & 0,47 & 54,16 & 9,5 & 20 & 0 & 0 & 3 & 0 & 217,5 & \\
\hline IM & & & & & 35 & 49,42 & & 1 & & \\
\hline Sost ss & 0,0000 & 0,0000 & 0,0000 & - & 0,0540 & 0,8893 & 0,1157 & 0,1602 & 0,0020 & 0,1526 \\
\hline Sost sl & 0,00 & 0,00 & 0,00 & - & 0,21 & 0,94 & 0,32 & 0,38 & 0,04 & 0,23 \\
\hline
\end{tabular}

\begin{tabular}{|c|c|c|c|c|c|c|c|c|c|c|}
\hline TERUEL & GRU & CA & CEHe & ZV & PM10 & TP & IM & VNPVP & DU & SM \\
\hline I & - & 122,7 & 66,24 & - & 2 & 5,17 & 1,51 & 0,46 & 41,27 & \\
\hline Io & 0,47 & 39,25 & 9,5 & 20 & 0 & 0 & 3 & 0 & 217,5 & \\
\hline IM & & & & & 35 & 50,93 & & 1 & & \\
\hline Sost ss & - & 0,0000 & 0,0000 & - & 0,9682 & 0,9030 & 0,0869 & 0,1230 & 0,0015 & 0,2975 \\
\hline Sost sl & - & 0,00 & 0,00 & - & 0,98 & 0,95 & 0,27 & 0,33 & 0,03 & 0,37 \\
\hline
\end{tabular}




\begin{tabular}{|c|c|c|c|c|c|c|c|c|c|c|}
\hline TOLEDO & GRU & CA & CEHe & ZV & PM10 & TP & IM & VNPVP & DU & SM \\
\hline I & 1,5 & 169,5 & 38,1 & - & 40 & 4,43 & 1,69 & 0,33 & 12,16 & \\
\hline Io & 0,47 & 35,62 & 9,49 & 20 & 0 & 0 & 3 & 0 & 217,5 & \\
\hline IM & & & & & 35 & 48,78 & & 1 & & \\
\hline Sost ss & 0,0000 & 0,0000 & 0,0000 & - & 0,0000 & 0,9216 & 0,1513 & 0,3401 & 0,0001 & 0,1766 \\
\hline Sost sl & 0,00 & 0,00 & 0,00 & - & 0,00 & 0,96 & 0,36 & 0,56 & 0,01 & 0,24 \\
\hline
\end{tabular}

\begin{tabular}{|c|c|c|c|c|c|c|c|c|c|c|}
\hline VALENCIA & GRU & CA & CEHe & ZV & PM10 & TP & IM & VNPVP & DU & SM \\
\hline I & 1,26 & 119,2 & 21,29 & 5,4 & 10 & 5,44 & 1,61 & 0,36 & 163 & \\
\hline Io & 0,47 & 46,83 & 9,49 & 20 & 0 & 0 & 3 & 0 & 217,5 & \\
\hline IM & & & & & 35 & 49,84 & & 1 & & \\
\hline Sost ss & 0,0000 & 0,0000 & 0,0000 & 0,0051 & 0,4455 & 0,8887 & 0,1193 & 0,2771 & 0,5366 & 0,2525 \\
\hline Sost sl & 0,00 & 0,00 & 0,00 & 0,06 & 0,65 & 0,94 & 0,32 & 0,50 & 0,72 & 0,35 \\
\hline
\end{tabular}

\begin{tabular}{|c|c|c|c|c|c|c|c|c|c|c|}
\hline VALLADOLID & GRU & CA & CEHe & ZV & PM10 & TP & IM & VNPVP & DU & SM \\
\hline I & 1,2 & 176 & 34,61 & 11,1 & 24 & 7,23 & 1,9 & 0,26 & 55,3 & \\
\hline Io & 0,48 & 46,87 & 9,46 & 20 & 0 & 0 & 3 & 0 & 217,5 & \\
\hline IM & & & & & 35 & 49,58 & & 1 & & \\
\hline Sost ss & 0,0000 & 0,0000 & 0,0000 & 0,1407 & 0,0095 & 0,8101 & 0,2641 & 0,5120 & 0,0041 & 0,1934 \\
\hline Sost sl & 0,00 & 0,00 & 0,00 & 0,35 & 0,08 & 0,89 & 0,49 & 0,70 & 0,05 & 0,29 \\
\hline
\end{tabular}

\begin{tabular}{|c|c|c|c|c|c|c|c|c|c|c|}
\hline VITORIA & GRU & CA & CEHe & ZV & PM10 & TP & IM & VNPVP & DU & SM \\
\hline I & 1,06 & 121,8 & 44,75 & 39,2 & 8 & 5,67 & 1,79 & 0,14 & 32,49 & \\
\hline Io & 0,48 & 90,52 & 9,51 & 20 & 0 & 0 & 3 & 0 & 217,5 & \\
\hline IM & & & & & 35 & 48,68 & & 1 & & \\
\hline Sost ss & 0,0000 & 0,3065 & 0,0000 & 0,0000 & 0,5961 & 0,8743 & 0,1997 & 0,8236 & 0,0008 & 0,3112 \\
\hline Sost sl & 0,00 & 0,53 & 0,00 & 0,00 & 0,76 & 0,93 & 0,42 & 0,90 & 0,02 & 0,40 \\
\hline
\end{tabular}

\begin{tabular}{|c|c|c|c|c|c|c|c|c|c|c|}
\hline ZAMORA & GRU & CA & CEHe & ZV & PM10 & TP & IM & VNPVP & DU & SM \\
\hline I & 1,3 & 207,4 & 31,61 & - & 31 & 8,33 & 1,79 & 0,35 & 57,98 & \\
\hline Io & 0,48 & 37,62 & 9,48 & 20 & 0 & 0 & 3 & 0 & 217,5 & \\
\hline IM & & & & & 35 & 50,78 & & 1 & & \\
\hline Sost ss & 0,0000 & 0,0000 & 0,0000 & - & 0,0003 & 0,7661 & 0,1997 & 0,2973 & 0,0049 & 0,1585 \\
\hline Sost sl & 0,00 & 0,00 & 0,00 & - & 0,01 & 0,87 & 0,42 & 0,52 & 0,06 & 0,24 \\
\hline
\end{tabular}

\begin{tabular}{|c|c|c|c|c|c|c|c|c|c|c|}
\hline ZARAGOZA & GRU & CA & CEHe & ZV & PM10 & TP & IM & VNPVP & DU & SM \\
\hline I & 1,33 & 116,1 & 34,29 & - & 124 & 4,43 & 2,09 & 0,25 & 24,07 & \\
\hline Io & 0,48 & 31,00 & 9,49 & 20 & 0 & 0 & 3 & 0 & 217,5 & \\
\hline IM & & & & & 35 & 49,94 & & 1 & & \\
\hline Sost ss & 0,0000 & 0,0000 & 0,0000 & - & 0,0000 & 0,9250 & 0,4020 & 0,5385 & 0,0004 & 0,2332 \\
\hline Sost sl & 0,00 & 0,00 & 0,00 & - & 0,00 & 0,96 & 0,61 & 0,72 & 0,02 & 0,29 \\
\hline
\end{tabular}


La figura 3 resume los datos de sostenibilidad media sensu stricto obtenidos para cada capital de provincia.

Figura 3. Distribución geográfica de la sostenibilidad urbana en España. Cada provincia está coloreada según el valor de sostenibilidad media que tiene la capital de provincia correspondiente.

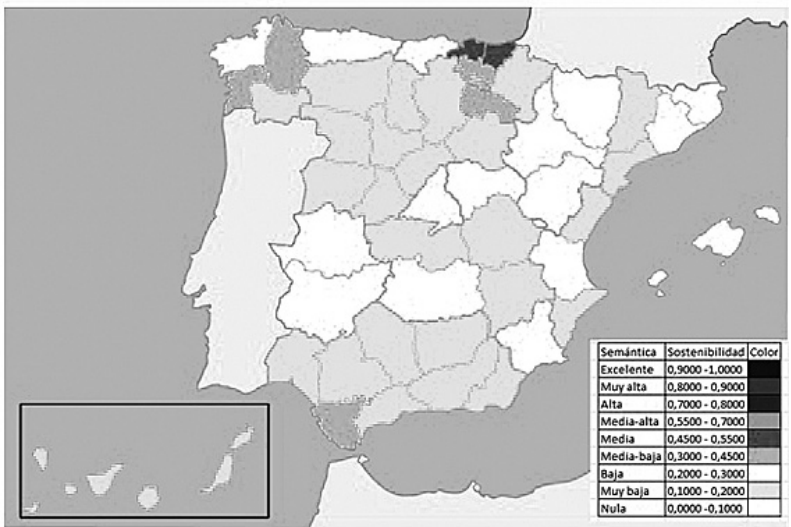

Fuente:Elaboración propia

La figura 4 es una gráfica-resumen con los valores de sostenibilidad media sensu stricto (azul) y sensu lato (rojo) de cada una de las capitales de provincia.

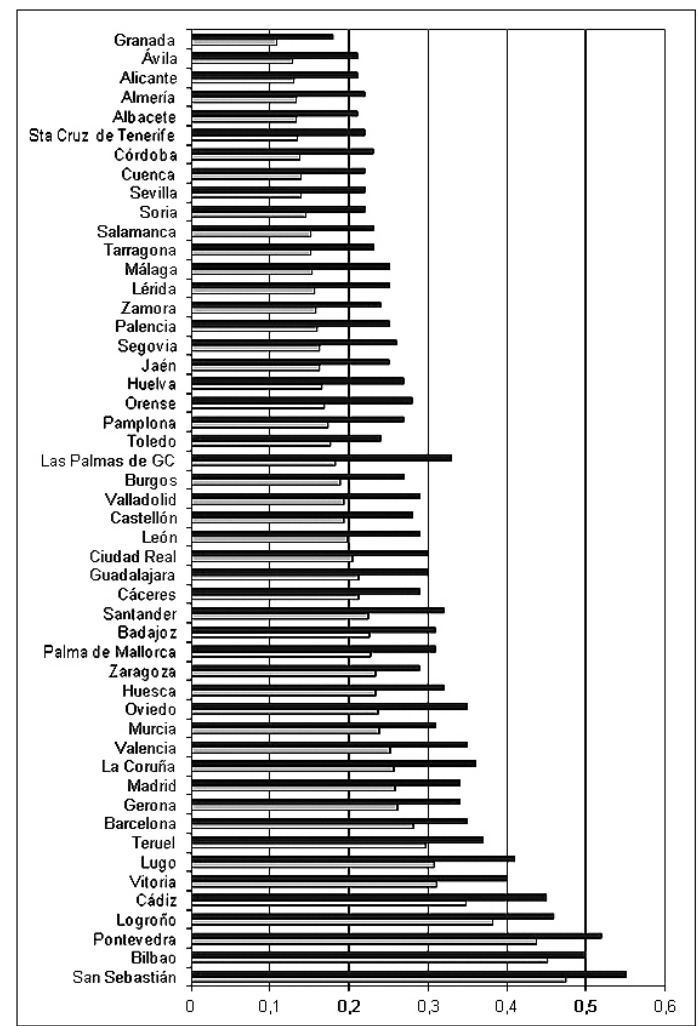


La figura 5 muestra los valores medios de sostenibilidad que obtiene cada indicador en el conjunto de las capitales de provincia.

Figura 5. Valores medios de sostenibilidad que alcanzan los diferentes indicadores analizados

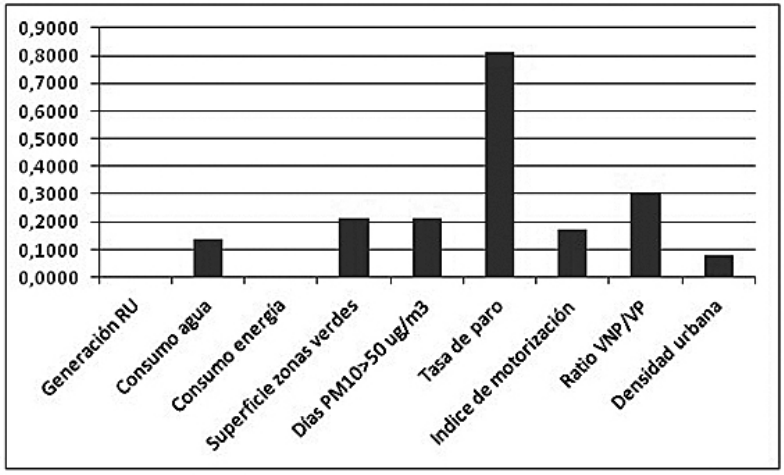

\section{CONCLUSIONES}

Puede observarse como la inmensa mayoría de las capitales de provincia de España (43 de 50) presentan una sostenibilidad baja (intervalo 0,2000-0,3000) o muy baja (intervalo $0,1000-0,2000$ ), y que ninguna supera el valor de sostenibilidad de 0,5000 (sostenibilidad sensu stricto).

Los indicadores analizados que presentan sistemáticamente valores de sostenibilidad muy bajos, incluso el valor 0,0000 , son los relacionados con el metabolismo urbano: Generación de residuos urbanos, Consumo de agua y Consumo de energía. Este resultado está en consonancia con los planteamientos establecidos en diferentes foros internacionales y que se resume en la frase "La batalla por la sostenibilidad se ganará o se perderá en las ciudades". Los resultados obtenidos inciden en el comportamiento de las ciudades como consumidoras insaciables de recursos, al tiempo que se abre la posibilidad de realizar unos planteamientos más ecológicos como base para futuras estrategias de sostenibilidad.

La generación de residuos urbanos y el consumo de energía alcanzan unos niveles claramente insostenibles. Se puede realizar la crítica fácil de que son una muestra de la sociedad consumista en la que estamos inmersos. La concienciación ciudadana es fundamental para reducir el valor de ambos indicadores, y que se aproximen a los valores considerados óptimos en este trabajo. Los ayuntamientos deben colaborar en todos los procesos que aumenten dicha concienciación. La Administración Central y la Autonómica deben legislar, y hacer que se cumpla la ley, para reducir tanto envase inútil que inunda los supermercados y las grandes superficies, que los cargamos los ciudadanos y termina siendo un Residuo Urbano. Hay que considerar que el mejor residuo es el que no se produce. Resulta totalmente absurdo que se considere un signo de desarrollo la producción de residuos urbanos. Es fundamental reducir en el origen. 
El consumo de agua presenta unos valores ligeramente mejores de sostenibilidad. La distribución geográfica de los valores de este indicador se aproxima bastante a la clásica división entre España Húmeda y España Seca. La mayoría de las ciudades españolas presentan valores bajos de sostenibilidad a causa de las características climatológicas de la mayor parte de la Península Ibérica. Puede parecer que el indicador está definido de tal modo que el valor Io resulta un tanto irreal. Consideramos que los postulados establecidos para el indicador Consumo de Agua ponen de manifiesto que el agua es un bien escaso. El valor de Io definido para cada capital resalta la escasez de agua que hay en gran parte del territorio español. Los ayuntamientos deben fomentar todas las medidas de ahorro y, sobre todo, dar ejemplo evitando malgastar el agua de forma indiscriminada. Es fundamental, también, que las EDAR realicen el tratamiento terciario que permita reutilizar el agua depurada ya sea para usos industriales como para usos agrícolas.

La mayoría de las ciudades españolas presentan un claro déficit de zonas verdes, $\mathrm{y}$, evidentemente, es el ayuntamiento quien debe establecer dichas zonas verdes. Hay que hacer una aclaración: las zonas verdes deben ser zonas verdes próximas a los ciudadanos; es absurdo considerar los grnades parques periurbanos como zonas verdes ya que, normalmente, están lejos de la mayoría de los ciudadanos.

Los niveles de contaminación atmosférica de las ciudades españolas está directamente relacionado con el tráfico rodado. El gran número de vehículos circulando es una de las causas de la presencia de partículas en la atmósfera (PM10) y contribuye a que este indicador presente valores bajos de sostenibilidad en la mayoría de las ciudades.

Relacionado con la morfología urbana y con el consumo de energía está el Índice de motorización. Los Ayuntamientos deben procurar que la ciudad resultante tras la correspondiente revisión del Plan de Ordenación Urbana sea una ciudad más compacta, que prime el transporte público y el uso de la bicicleta. La conclusión a la que se llega es que hay demasiados vehículos privados moviéndose casi todos a la vez.

Las viviendas no principales (VNP) contribuyen a que la ciudad sea algo menos compacta. Existen demasiadas VNP en las ciudades españolas. En algunos casos pueden ser el resultado de la pérdida de población por migración, en muchos casos son el resultado de aspectos relacionados con el turismo; en otros muchos casos son el resultado de una especulación urbanística que nadie ha sabido o querido parar. $\mathrm{La}$ propia administración pública favorece este crecimiento del número de viviendas. Sólo por citar un ejemplo, el Plan de Ordenación del Territorio de Andalucía admite un crecimiento máximo de la población del $30 \%$ cada 8 años que equivale a decir que la población andaluza se duplicará en unos 20 años y, por tanto, habrá que construir las correspondientes viviendas. ¿Alguien en su sano juicio cree que en 20 años se va a duplicar la población andaluza?

La Densidad urbana es otro indicador con valores muy bajos de sostenibilidad. Este indicador está relacionado con la morfología urbana y, en cierta manera, mide el grado de compacidad de la ciudad. Se habla mucho de la ciudad compacta, mediterránea, etc., pero la realidad es que muy pocas capitales de provincia son realmente ciudades compactas, tal y como se deduce de los valores de sostenibilidad obteni- 
dos. No conocemos ningún estudio que deduzca la densidad de viviendas a partir de unos postulados objetivos, ni que defina claramente qué se entiende por ciudad compacta. Los trabajos previos realizados por los autores mostraban cómo los barrios de Sevilla que se relacionan intuitivamente con una ciudad compacta y mediterránea alcanzan valores bajos de sostenibilidad para el indicador Densidad Urbana. Parece como si el concepto de ciudad compacta y mediterránea no cuadrara con la ciudad considerada "típicamente" mediterránea. Hay que plantear claramente que los ayuntamientos deben procurar que la ciudad resultante tras la correspondiente revisión del Plan de Ordenación Urbana sea una ciudad más compacta. Hay que estudiar con más profundidad cual es la densidad de viviendas que produce un máximo de sostenibilidad. La consideración realizada en este trabajo de 75 viv/Ha es un valor máximo entre todos los valores publicados.

La tasa de paro parece muy sostenible. No hay que olvidar que los datos son anteriores a la actual crisis económica y la tasa de paro era muy inferior a la actual. Una estimación actualizando datos produce un valor medio de sostenibilidad aproximado de 0,2000, y bajaría aún más el valor de sostenibilidad medio de las capitales de provincia.

\section{BIBLIOGRAFÍA}

ECOLOGISTAS EN ACCIÓN. Informe La calidad del aire en el estado español durante 2008.

INSTITUTO NACIONAL DE ESTADÍSTICA.

OBSERVATORIO DE SOSTENIBILIDAD DE ESPAÑA. "Sostenibilidad local: una aproximación urbana y rural".

RODRÍGUEZ MELLADO, JOSEFA MARÍA y RIVERO PALLARÉS, FRANCISCO. 2010a. Definición y aplicación de una función de sostenibilidad. Aplicación al análisis de la sostenibilidad urbana. $7^{\circ}$ Congreso Virtual de Desarrollo Sostenible. 2010.

RODRÍGUEZ MELLADO, JOSEFA MARÍA y RIVERO PALLARÉS, FRANCISCO. 2010b. Análisis de la Sostenibilidad de la ciudad de Sevilla. $10^{\circ}$ Congreso Nacional de Medio Ambiente. 22 al 26 de Noviembre de 2010.

VARIOS AUTORES. Guía para la elaboración de estudios del medio físico. Ministerio de Obras Públicas y Transportes. 1992. 